Bull. Korean Math. Soc. 51 (2014), No. 6, pp. 1851-1861

http://dx.doi.org/10.4134/BKMS.2014.51.6.1851

\title{
A NOTE ON ZERO DIVISORS IN $w$-NOETHERIAN-LIKE RINGS
}

\author{
Hwankoo Kim, Tae In Kwon, and Min Surp Rhee
}

\begin{abstract}
We introduce the concept of $w$-zero-divisor ( $w$-ZD) rings and study its related rings. In particular it is shown that an integral domain $R$ is an SM domain if and only if $R$ is a $w$-locally Noetherian $w$-ZD ring and that a commutative ring $R$ is $w$-Noetherian if and only if the polynomial ring in one indeterminate $R[X]$ is a $w$-ZD ring. Finally we characterize universally zero divisor rings in terms of $w$-ZD modules.
\end{abstract}

\section{Introduction}

As natural generalizations of Noetherian rings, various important classes of rings were defined and studied in the literature. Let $R$ be a commutative ring with identity. An ideal $Q$ of $R$ is strongly primary if $Q$ is primary and contains a power of its radical. The ring $R$ is said to be Laskerian if each ideal of $R$ is a finite intersection of primary ideals, and $R$ is said to be strongly Laskerian if each ideal of $R$ is a finite intersection of strongly primary ideals. These rings are investigated systemically in [8]. Evans in [3] showed that a Laskerian ring is what he calls a ZD-ring (for zero-divisor ring), which is defined as follows. A ring $R$ is a $Z D$ ring if the set $\mathscr{Z}_{R}(R / I)$ of zero divisors on the $R$-module $R / I$ is a finite union of prime ideals for each ideal $I$ of $R$. A nonzero $R$-module $M$ is called a zero divisor module (ZD module), if $\mathscr{Z}_{R}(M / N)$ is a finite union of prime ideals for all submodules $N$ of $M$. It was shown in [6] that a Laskerian ring has Noetherian spectrum. Thus we have the following implications:

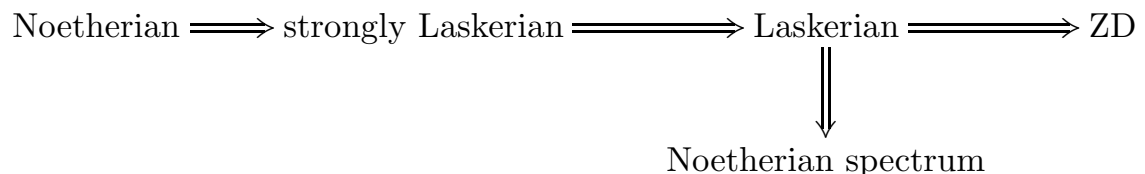

Received December 2, 2013; Revised March 23, 2014

2010 Mathematics Subject Classification. Primary 13A15; Secondary 13E99, 13 F05.

Key words and phrases. zero divisor, zero divisor ring, zero divisor module, universally zero divisor ring, w-operation. 
In [10], Heinzer and Ohm proved that $R$ is Noetherian if $R[X]$ is a ZD-ring, and hence the conditions Noetherian, strongly Laskerian, Laskerian and ZD are equivalent in $R[X]$.

Based on a star-operation theoretical approach, Wang and McCasland introduced the concept of strong Mori domains (SM domains), which generalize Noetherian domains. Since then, this concept has been extended to commutative rings (which is so-called a $w$-Noetherian ring) and studied extensively. Thus a natural question arises as follows: If we define the corresponding rings with respect to the $w$-operation, are the properties and their implications of original rings preserved in the corresponding ones? In this paper, we explore this question.

We first introduce some definitions and notations. Let $R$ be an integral domain with quotient field $K$. For a nonzero fractional ideal $I$ of $R$, set $I^{-1}:=$ $\{x \in K \mid x I \subseteq R\}, I_{v}:=\left(I^{-1}\right)^{-1}, I_{t}:=\bigcup\left\{J_{v} \mid J \subseteq I\right.$ finitely generated subideal of $I\}$, and $I_{d}:=I$. An ideal $J$ of $R$ is called a $G V$-ideal, denoted by $J \in \mathrm{GV}(R)$, if $J$ is a finitely generated ideal of $R$ with $J^{-1}=R$. For a torsion-free $R$-module $M$, Wang and McCasland defined the $w$-envelope of $M$ as $M_{w}:=\left\{x \in M \otimes_{R} K \mid J x \subseteq M\right.$ for some $\left.J \in \operatorname{GV}(R)\right\}$ ([19], cf., [13]). A torsion-free $R$-module is called a $w$-module (or semidivisorial module) if $M_{w}=M$.

The above concepts can be extended to commutative rings ([22]). Let $J$ be a finitely generated ideal of a commutative $\operatorname{ring} R$. In [22] $J$ is called a GlazVasconcelos ideal or a $G V$-ideal if the natural homomorphism $\varphi: R \rightarrow J^{b}:=$ $\operatorname{Hom}_{R}(J, R)$ is an isomorphism. Note that the set $\operatorname{GV}(R)$ of GV-ideals of $R$ is a multiplicative system of ideals of $R$. It is obvious that a finitely generated ideal $J$ of $R$ is a GV-ideal if and only if $\operatorname{Hom}_{R}(R / J, R)=0$ and $\operatorname{Ext}_{R}^{1}(R / J, R)=0$.

Let $M$ be an $R$-module with the injective envelope $E(M)$. Define

$$
\operatorname{tor}_{\mathrm{GV}}(M)=\{x \in M \mid J x=0 \text { for some } J \in \mathrm{GV}(R)\} \text {. }
$$

Then $\operatorname{tor}_{\mathrm{GV}}(M)$ is a submodule of $M$ and is called a $G V$-torsion submodule of $M . M$ is called a $G V$-torsion (resp., $G V$-torsion-free) module if $\operatorname{tor}_{\mathrm{GV}}(M)=$ $M$ (resp., $\operatorname{tor}_{\mathrm{GV}}(M)=0$ ). A GV-torsion-free $R$-module $M$ is called a $w$-module if $\operatorname{Ext}_{R}^{1}(R / J, M)=0$ for any $J \in \mathrm{GV}(R)$. For any $\mathrm{GV}$-torsion-free module $M$,

$$
M_{w}=\{x \in E(M) \mid J x \subseteq M \text { for some } J \in \mathrm{GV}(R)\}
$$

is a $w$-submodule of $E(M)$ containing $M$ and is called the $w$-envelope of $M$. It is clear that a GV-torsion-free module $M$ is a $w$-module if and only if $M_{w}=M$. An $R$-module $M$ is said to be of $w$-finite type if there exists a finitely generated submodule $B$ of $M$ such that $M_{w}=B_{w}$. A prime ideal which is a $w$-ideal is called a prime $w$-ideal. We denote by $w$-Spec $(R)$ the set of prime $w$-ideals of $R$. A $w$-ideal is called a maximal $w$-ideal of $R$ if it is maximal among integral $w$ ideals of $R$. It is known that a maximal $w$-ideal is a prime ideal 22 , Proposition $3.8]$. 
A commutative ring $R$ is said to be $w$-Noetherian if $R$ satisfies the ACC on its $w$-ideals ([22, Definition 4.1]). An integral domain is called a strong Mori domain (SM domain) if it is $w$-Noetherian. A commutative ring $R$ is said to be $w$-locally Noetherian if $R_{\mathfrak{m}}$ is Noetherian for every maximal $w$-ideal $\mathfrak{m}$ of $R$. A commutative ring $R$ is called a $D W$ ring if every ideal of $R$ is a $w$-ideal. In [18, Theorem 3.8], it was shown that $R$ is a DW ring if and only if every maximal ideal of $R$ is a $w$-ideal.

Any undefined terminology is standard, as in [5].

\section{Main results}

Let $M$ be an $R$-module. A prime ideal $\mathfrak{p}$ of $R$ is called an associated prime ideal of $M$ if there exists $x \in M \backslash\{0\}$ such that $\mathfrak{p}$ is a prime ideal minimal over $\operatorname{ann}(x)$. We denote by $\operatorname{Ass}(M)$ the set of associated prime ideals of $M$. It is clear that if $M \neq 0$, then $\operatorname{Ass}(M)$ is nonempty. It was shown in [20, Lemma 3.1 ] that if $\mathfrak{p} \in \operatorname{Ass}(M)$ for a $\mathrm{GV}$-torsion-free $R$-module, then $\mathfrak{p}$ is a $w$-ideal of $R$.

Theorem 2.1 ([20, Theorem 3.9, Theorem 3.17, and Corollary 3.18]). Let $R$ be a w-Noetherian ring and let $M$ be a GV-torsion-free module. Then we have

(a) $\mathscr{Z}(M)=\bigcup_{\mathfrak{p} \in \operatorname{Ass}(M)} \mathfrak{p}$;

(b) in addition, if $M$ is a w-finite type w-module, then $\operatorname{Ass}(M)$ is a finite set;

(c) if $I$ is a $w$-ideal of $R$, then there are only finitely many prime ideals of $R$ minimal over $I$.

We say that a ring $R$ is a $w-Z D$ ring if the set of zero divisors on the $R$ module $R / A$ is a finite union of prime $w$-ideals for each $w$-ideal $A$ of $R$. Then it follows from Theorem 2.1 that if $R$ is a $w$-Noetherian ring, then $R$ is a $w$-ZD ring.

Given an ideal $I$ of a commutative ring $R$, a prime ideal $\mathfrak{p}$ is said to be a weak Bourbaki associated prime ideal for $I$ (or simply a $\mathrm{B}_{\mathrm{w}}$-prime) if $\mathfrak{p}$ is minimal over $\left(I:_{R} x\right)$ for some $x \in R \backslash I$. Moreover, if $I$ is a $w$-ideal of $R$, then $\mathfrak{p}$ is a $w$-ideal of $R$ (cf. [7, Proposition 1.1]). A prime ideal $\mathfrak{p}$ of $R$ such that $\mathfrak{p}$ is maximal with respect to the property of being contained in $\mathscr{Z}_{R}(R / I)$ is called a maximal $N$-prime (for Nagata-prime) of $I$. As pointed out in [10], such a prime ideal contains $I$ and $\mathscr{Z}_{R}(R / I)$ is the union of the maximal $\mathrm{N}$-primes of $I$. Moreover, if $I$ is a $w$-ideal of $R$, then such a prime ideal is also a $w$-ideal of $R$.

We say that a commutative ring $R$ is $w$-Laskerian if each proper $w$-ideal of $R$ may be expressed as a finite intersection of primary $w$-ideals of $R$. Then it was shown in [14, Theorem 2.7] that a domain $R$ is an SM domain if and only if $R$ is a $w$-locally Noetherian and $w$-Laskerian domain. It was also shown in [10, Proposition] that a commutative ring $R$ is Noetherian if and only if $R$ is a 
locally Noetherian ZD-ring. Thus the following result, which is a $w$-analogue of [10, Proposition], strengthens [14, Theorem 2.7].

Proposition 2.2. Let $R$ be a domain. Then $R$ is an $S M$ domain if and only if $R$ is a $w$-locally Noetherian $w$-ZD ring.

Proof. By [4, Theorem 4.5] it suffices to show that every $w$-ideal $I$ of $R$ has only finitely many $\mathrm{B}_{\mathrm{w}}$-primes. If $\mathfrak{p}$ is a $\mathrm{B}_{\mathrm{w}}$-prime of $I$, then $\mathfrak{p}$ is contained in a maximal N-prime of $I$. Since $R$ is a $w$-ZD ring, $I$ has only a finite number of maximal $\mathrm{N}$-primes, say $\mathfrak{q}_{1}, \ldots, \mathfrak{q}_{n}$. Moreover, since $\mathfrak{p}$ is a $\mathrm{B}_{\mathrm{w}}$-prime of $I$ in $R$ and $\mathfrak{p} \subset \mathfrak{q}_{i}$, we have that $\mathfrak{p} R_{\mathfrak{q}_{i}}$ is a $\mathrm{B}_{\mathrm{w}}$-prime of $I R_{\mathfrak{q}_{i}}$ ([9, Proposition 1.2]). Since $R_{\mathfrak{q}_{i}}$ is Noetherian, $I R_{\mathfrak{q}_{i}}$ has only finitely many $\mathrm{B}_{\mathrm{w}}$-primes. Hence $I$ has only finitely many $\mathrm{B}_{\mathrm{w}}$-primes.

In [22, Proposition 2.6], it was shown that for a $\mathrm{GV}$-torsion-free $R$-module $M$ and a directed family $\left\{M_{i} \mid i \in \Gamma\right\}$ of $w$-submodules of $M, \bigcup_{i \in \Gamma} M_{i}$ is a $w$-submodule of $M$.

A commutative ring $R$ is said to be strongly $w$-Laskerian if $R$ is $w$-Laskerian and every primary $w$-ideal of $R$ contains a power of its radical.

Lemma 2.3. Let $R \subseteq T$ be an extension of commutative rings such that $(I T)_{w} \cap R=I_{w}$ for every ideal $I$ of $R$. If $T$ is $w$-Laskerian, strongly $w$ Laskerian, or $w-Z D$, then so is $R$.

Proof. For $w$-Laskerian or strongly $w$-Laskerian, this is trivial. For the $w$-ZD case, consider a $w$-ideal $A$ of $R$. Let $\mathscr{C}:=\{C \mid C$ is a $w$-ideal of $T$ such that $C \cap R=A\}$. Then by hypothesis $\mathscr{C}$ is nonempty. Thus by Zorn's lemma there is a maximal element in $\mathscr{C}$, say $B$. Then $\mathscr{Z}_{R}(R / A)=\mathscr{Z}_{T}(T / B) \cap R$. Indeed, if $r \in \mathscr{Z}_{T}(T / B) \cap R$, then there exists $t \in T$ such that $r t \in B$ and $t \notin B$. Thus $t \in\left(B:_{T}(r)\right)$ and so $B \subsetneq\left(B:_{T}(r)\right)$. Note that $\left(B:_{T}(r)\right)$ is a $w$-ideal. Indeed, let $J \in G V(T)$ and $x \in T$ such that $x J \subseteq\left(B:_{T}(r)\right)$. Then $x r J \subseteq B$. Hence, since $B$ is a $w$-ideal, it follows that $x r \in B$, i.e., $x \in\left(B:_{T}(r)\right)$. Thus, $\left(B:_{T}(r)\right)$ is a $w$-ideal. Hence $\left(B:_{T}(r)\right) \cap R \supsetneq A$. So there is $r^{\prime} \in\left(B:_{T}(r)\right) \cap R$ but $r^{\prime} \notin A$. Therefore $r r^{\prime} \in A$ and hence $r \in \mathscr{Z}_{R}(R / A)$. The other inclusion is straightforward.

Let $R$ be an integral domain. Recall that $R$ is a strong Mori domain if it satisfies the ascending chain condition on integral $w$-ideals. The strong Mori property can be extended to the spectrum of integral domains. We define $R$ to have strong Mori spectrum if it satisfies the descending chain condition on the sets of the form $W(I):=\{P \in w$-Spec $(R) \mid I \subseteq P\}$, where $I$ runs over $w$ ideals of $R$ (or equivalently, the induced topology on $w$-Spec $(R)$ by the Zariski topology on $\operatorname{Spec}(R)$ is Noetherian). Note that for every nonzero ideal $I$ of $R$, $V(I) \cap w$-Spec $(R)=W\left(I_{w}\right)$, where $V(I):=\{P \in \operatorname{Spec}(R) \mid I \subseteq P\}$ is a closed set in $\operatorname{Spec}(R)$. For all $w$-ideals $I, J$ of $R$, we have $W(I) \cup W(J)=W(I \cap J)$ and $W(I) \cap W(J)=W\left((I+J)_{w}\right)$. This concept extends that of Noetherian spectrum and certainly an integral domain with Noetherian spectrum has 
strong Mori spectrum. In the case when $w=d$ (for example, Prüfer domains or one-dimensional domains), the notions of Noetherian spectrum and strong Mori spectrum coincide.

The following two lemmas can be proved easily from the proofs of $[1$, Lemma 3.7 and Lemma 3.8] by substituting the $w$-operation for both the $v$-operation and the $t$-operation.

Lemma 2.4. Let $R$ be an integral domain. Then the following are equivalent.

(1) Each prime $w$-ideal of $R$ is the radical of a $w$-finite type ideal.

(2) Each radical $w$-ideal of $R$ is the radical of a $w$-finite type ideal.

(3) $R$ satisfies the ascending chain condition on radical w-ideals.

Lemma 2.5. Let $R$ be an integral domain satisfying the ascending chain condition on radical $w$-ideals. Then

(1) Any radical $w$-ideal of $R$ is the intersection of a finite number of prime $w$-ideals.

(2) If $I$ is a $w$-ideal of $R$, then the set of minimal prime (w-)ideals of $I$ is finite.

In the following, we give some characterizations of integral domains with strong Mori spectrum. The proof of this result closely follows the proof in the Mori spectrum case [2, Proposition 2.1]. Yet for the sake of completeness we include a proof. Note that $(\sqrt{I})_{w}=\sqrt{I_{w}}$ for each ideal $I$ of $R$ ([19, Proposition $2.4])$.

Theorem 2.6. Let $R$ be an integral domain. Then the following conditions are equivalent.

(1) $R$ has strong Mori spectrum.

(2) $R$ satisfies the ascending chain condition on radical $w$-ideals.

(3) For every nonzero ideal I of $R, \sqrt{I_{w}}=\sqrt{J_{w}}$ for some finitely generated subideal $J$ of $I$.

(4) Each radical $w$-ideal of $R$ is the radical of a $w$-finite type ideal.

(5) Each prime $w$-ideal of $R$ is the radical of a $w$-finite type ideal.

(6) $R$ satisfies the ascending chain condition on prime $w$-ideals and each proper $w$-ideal has only finitely many minimal ( $w$-)primes.

(7) $R$ satisfies the ascending chain condition on radicals of $w$-ideals.

Proof. $(1) \Leftrightarrow(2)$ This follows from the fact that for every $w$-ideals $I, J$ of $R$, $W(J) \subseteq W(I)$ if and only if $\sqrt{J} \supseteq \sqrt{I}$.

$(2) \Leftrightarrow(3) \Leftrightarrow(4) \Leftrightarrow(5)$ These follow from Lemma 2.4 and the fact that $\sqrt{I_{w}}$ is a radical $w$-ideal of $R$.

$(2) \Rightarrow(6)$ Lemma 2.5.

$(6) \Rightarrow(2)$ Let $\left\{I_{k}\right\}_{k \geq 1}$ be a properly ascending chain of radical $w$-ideals, and let $I_{1}=P_{1} \cap \cdots \cap P_{r}$ for some prime $\left(w\right.$-)ideals $P_{1}, \ldots, P_{r}$. For $i=1, \ldots, r$, set $I_{k, i}:=\sqrt{\left(I_{k}+P_{i}\right)_{w}}$. We get a family of $r$ ascending chains of radical $w$-ideals $\left\{I_{k, i}\right\}_{k \geq 1}$ with $I_{1, i}=P_{i}$ for every $i=1, \ldots, r$. Assume that all these chains 
stop, and let $n$ be a positive integer such that $I_{n, i}=I_{n+1, i}$ for all $i=1, \ldots, r$. Choose any $x \in I_{n+1}$. Since $I_{n+1} \subseteq I_{n, i}$, there exists an integer $m \geq 1$ such that $x^{m} \in\left(I_{n}+P_{i}\right)_{w}$ for every $i=1, \ldots, r$. Therefore we obtain

$$
\begin{aligned}
x^{m r} & \in\left(I_{n}+P_{1}\right)_{w} \cdots\left(I_{n}+P_{r}\right)_{w} \\
& \subseteq\left(\left(I_{n}+P_{1}\right) \cdots\left(I_{n}+P_{r}\right)\right)_{w} \\
& \subseteq\left(I_{n}+P_{1} \cdots P_{r}\right)_{w} \\
& \subseteq\left(I_{n}+I_{1}\right)_{w} \\
& =I_{n} .
\end{aligned}
$$

Hence $x \in I_{n}$, and so $I_{n}=I_{n+1}$, a contradiction. Say that $\left\{I_{k, 1}\right\}_{k \geq 1}$ is a properly ascending chain of radical $w$-ideals. Then $P_{1}=I_{1,1} \subset I_{2,1} \subset I_{3,1} \subset$ $\cdots$. By the same argument applied to the chain of radical $w$-ideals $\left\{I_{k, 1}\right\}_{k \geq 2}$, there exists a prime $w$-ideal $Q_{1}$ such that $P_{1} \subset Q_{1}$. By iterating this process, we get a properly ascending chain of prime $w$-ideals.

$(2) \Leftrightarrow(7)$ This can be shown using (3).

In [6, Theorem 4], it was shown that every Laskerian ring has Noetherian spectrum. The following result is the $w$-theoretic analogue of [6, Theorem 4].

Theorem 2.7. A w-Laskerian domain has strong Mori spectrum.

Proof. By Theorem 2.6, it is enough to prove that the ascending chain condition on prime $w$-ideals is satisfied in $R$. Let $R$ be a $w$-Laskerian ring and assume that $R$ does not have strong Mori spectrum. Then there exists an infinite strictly ascending sequence

$$
P_{1} \subset P_{1}^{\prime} \subset P_{2} \subset P_{2}^{\prime} \subset \cdots
$$

of proper prime $w$-ideals of $R$. Since every localization of a $w$-Laskerian domain at a prime $w$-ideal is Laskerian, as in the proof of [6, Theorem 4], we can find $w$-ideals $Q_{1}, \ldots, Q_{n}, A_{n}, B_{n}$ of $R$ and elements $x_{1}, x_{2}, \ldots, x_{n}$ of $R$ with the following properties.

(i) $Q_{i}$ is $P_{i}$-primary for each $i$, and $A_{n}=Q_{1} \cap \cdots \cap Q_{n}$.

(ii) For $1 \leq i \leq n, x_{i} \in \bigcap_{j \neq i} Q_{j}$ and $x_{i} \notin Q_{i}$.

(iii) $\left(x_{1}, \ldots, x_{n}\right) \subseteq B_{n}, A_{n} \nsubseteq B_{n}$ and each belonging prime of $B_{n}$ is contained in $P_{n}^{\prime}$.

Now we define $A=\bigcap_{i=1}^{\infty} Q_{i}$. Then the proof of [6, Theorem 4] shows that $A$ admits no representation as a finite intersection of primary $w$-ideals. Therefore $R$ is not $w$-Laskerian, as desired.

If $R$ is an integral domain, we set $R\{X\}:=R[X]_{N_{v}}$, where $N_{v}:=\{f \in$ $\left.R[X] \mid c(f)_{v}=R\right\}$, a multiplicative set in $R[X](c(f)$ is the ideal of $R$ generated by the coefficients of $f) . R\{X\}$ is called the $t$-Nagata ring of $R$ ([11]).

Proposition 2.8. Let $R$ be a domain with $w-\operatorname{dim}(R)=1$. Then the following statements are equivalent. 
(1) $R$ is a $w-Z D$ domain.

(2) $R$ has strong Mori spectrum.

(3) $R$ is w-Laskerian.

(4) $R\{X\}$ is a $Z D$ domain.

Proof. (1) $\Rightarrow(3)$ Let $r$ be a nonzero nonunit of $R$. Let $I:=(r)$. By hypothesis, we have that there are only a finite number of prime $w$-ideals minimal over $I$. Thus any nonzero nonunit of the domain $R$ with $w$-dim $(R)=1$ belongs to only a finite number of prime $w$-ideals of $R$. Hence $R$ is $w$-Laskerian.

(3) $\Rightarrow(2)$ Theorem 2.7 .

$(2) \Rightarrow(1)$ Let $I$ be a $w$-ideal of $R$. Then note that $\mathscr{Z}_{R}(R / I)$ is a union of prime $w$-ideals of $R$ ([12, Theorem 2]). Now the assertion follows from Theorem $2.6(5)$.

(4) $\Rightarrow(1)$ Since $R\{X\}$ is a faithfully flat $R$-module, this follows from Lemma 2.3 and the fact that $R\{X\}$ is a DW domain.

$(1) \Rightarrow(4)$ Note first that the maximal ideals of $R\{X\}$ are the extensions of the maximal $w$-ideals of $R$, and that any nonzero ideal of $R\{X\}$ is contained in only finitely many maximals (by the strong Mori spectrum in $R$ ). So we may assume $(R, \mathfrak{m})$ is $w$-local. Let $A$ be a nonzero ideal of $R\{X\}$. If $\mathfrak{m}\{X\} \subseteq \mathscr{Z}_{R\{X\}}(R\{X\} / A)$, then they are equal, and we are done. Otherwise $\mathscr{Z}_{R\{X\}}(R\{X\} / A) \cap R=(0)$. But then we may localize at the nonzero elements of $R$, and transfer the problem to a localization of a polynomial ring over the quotient field of $R$, where the result is clear.

In [10, Theorem], it was shown that a commutative ring $R$ is Noetherian if and only if the polynomial ring $R[X]$ in one indeterminate $X$ is a ZD-ring. Now we explore the $w$-theoretic analogue of [10, Theorem]. To do so, we first recall the following easy observation $[15$, p. 363]. Let $A$ be an $R$-submodule of the total quotient ring $T(R)$ of $R$. Set $A^{-1}:=\{u \in T(R) \mid u A \subseteq R\}$. Define $\xi: A^{-1} \rightarrow A^{b}=\operatorname{Hom}_{R}(A, R)$ by $\xi(u)(a)=a u, a \in A, u \in A^{-1}$. Then $\xi$ is a homomorphism. It is easy to see that if $I$ is a regular ideal of $R$, then $\xi: I^{-1} \rightarrow I^{b}$ is an isomorphism. Thus we have that for a finitely generated regular ideal $J$ of $R, J \in \mathrm{GV}(R)$ if and only if $J^{-1}=R$. Moreover, if $I$ is a regular ideal of $R$, then $I$ is a $w$-ideal if and only if $J x \subseteq I$ implies that $x \in I$, where $J$ is a regular GV-ideal of $R$ and $x \in R$.

Let $I$ be an ideal of $R[X]$. Then we denote by $c(I)$ the ideal of $R$ generated by the coefficients of all polynomials in $I$. In [21, Corollary 2.5], it was shown that if $J \in \mathrm{GV}(R[X])$, then $c(J) \in \mathrm{GV}(R)$, and hence there exists a non-zerodivisor $f \in J$ of $R[X]$ such that $c(f)_{w}=R$. Therefore, every GV-ideal of $R[X]$ is regular.

Theorem 2.9. $R$ is $w$-Noetherian if and only if $R[X]$ is a $w-Z D$ ring.

Proof. Suppose that $R$ is not $w$-Noetherian. Then there exists a strictly ascending chain $(0) \subset\left(a_{1}\right)_{w} \subset\left(a_{1}, a_{2}\right)_{w} \subset \cdots \subset\left(a_{1}, \ldots, a_{n}\right)_{w} \subset \cdots$ of $w$-ideals of $R$. Let $f_{0}=X, f_{1}=1+X, \ldots, f_{i}=1+f_{0} f_{1} \cdots f_{i-1}, \ldots$ We wish to 
show that the $w$-ideal $I:=\left(a_{1} f_{1}, a_{2} f_{1} f_{2}, \ldots, a_{n} f_{1} \cdots f_{n}, \ldots\right)_{W}$ in $R[X]$ has an infinite number of maximal N-primes, where $W$ is the $w$-operation on $R[X]$, and hence has the property that $\mathscr{Z}_{R[X]}(R[X] / I)$ is not a finite union of prime $w$-ideals.

Claim. Each $f_{i} \in \mathscr{Z}_{R[X]}(R[X] / I)$.

Since $f_{1}$ is not a zero divisor, we have that $I \subseteq\left(f_{1}\right)$. Since $f_{1}$ is a monic polynomial of positive degree in $R[X]$, it follows that $I \cap R=(0)$. Hence $a_{1} \notin I$, so $a_{1} f_{1} \in I$ implies that $f_{1} \in \mathscr{Z}_{R[X]}(R[X] / I)$. Similarly, to show that $f_{n} \in \mathscr{Z}_{R[X]}(R[X] / I)$, we wish to show that $a_{n} f_{1} \cdots f_{n-1} \notin I$. Consider the residue $\operatorname{ring} \bar{R}:=R /\left(a_{1}, \ldots, a_{n-1}\right)_{w}$. For $f \in R[X]$ (resp., $I \subseteq$ $R[X]$ ), we denote by $\bar{f}$ (resp., $\bar{I}$ ) the image of $f$ (resp., $I$ ) in $\bar{R}[X]$. Thus by above remarks, for an ideal $J$ of $R[X], J \in \operatorname{GV}(R[X])$ if and only if $\bar{J} \in \operatorname{GV}(\bar{R}[X])$. Then $\bar{I}=\left(\overline{a_{n}} \overline{f_{1}} \ldots \overline{f_{n}}, \overline{a_{n+1}} \overline{f_{1}} \cdots \overline{f_{n+1}}, \ldots\right)_{\bar{W}}$, where $\bar{W}$ is the $w$-operation on $\bar{R}[X]$. It will suffice to show that $\overline{a_{n}} \overline{f_{1}} \cdots \overline{f_{n-1}} \notin \bar{I}$; and since $\overline{f_{1}} \cdots \overline{f_{n-1}}$ is a monic polynomial in $\bar{R}[X]$, this is equivalent to showing that $\overline{a_{n}} \notin\left(\overline{a_{n}} \overline{f_{n}}, \overline{a_{n+1}} \overline{f_{n} f_{n+1}}, \ldots\right) \bar{W} \subseteq\left(\overline{f_{n}}\right)$. Since $\overline{f_{n}}$ is a monic polynomial of positive degree in $\bar{R}[X]$, we have that $\left(\overline{f_{n}}\right) \cap \bar{R}=(0)$. Thus $\overline{a_{n}} \notin\left(\overline{a_{n}} \overline{f_{n}}, \overline{a_{n+1}} \overline{f_{n} f_{n+1}}, \ldots\right) \bar{W}$; hence we have proved $f_{n} \in \mathscr{Z}_{R[X]}(R[X] / I)$.

Consider now $f_{i}$ and $f_{j}$ for $i \neq j$. Clearly no prime $w$-ideal of $R[X]$ contains both $f_{i}$ and $f_{j}$. Since each $f_{i} \in \mathscr{Z}_{R[X]}(R[X] / I)$ and hence is in some maximal $\mathrm{N}$-prime of $I$, it then follows that $I$ has infinitely many maximal $\mathrm{N}$-primes.

Corollary 2.10. Let $R$ be a commutative ring. Then the following statements are equivalent.

(1) $R[X]$ is a $w-Z D$ ring.

(2) $R[X]$ is $w$-Laskerian.

(3) $R[X]$ is strongly $w$-Laskerian.

(4) $R[X]$ is $w$-Noetherian.

We say that a nonzero $\mathrm{GV}$-torsion-free $R$-module $M$ is called a $w$-zero divisor module ( $w$-ZD module), if $\mathscr{Z}_{R}(M / N)$ is a finite union of prime $w$-ideals for all $w$-submodules $N$ of $M$. Recall that a commutative $\operatorname{ring} R$ is a universally zero-divisor ring (UZD ring) if every $R$-module is a ZD $R$-module. In $[17$, Proposition 1], it was shown that a commutative ring $R$ is a UZD ring if and only if the union of any family of prime ideals of $R$ is the union of a finite number of prime ideals of $R$ (not necessarily belonging to the same family). In the following, we give a new characterization of a UZD ring.

Theorem 2.11. Let $R$ be a commutative ring. Then the following are equivalent.

(1) $R$ is a UZD ring.

(2) Every $G V$-torsion-free $R$-module is a $w$-ZD R-module.

(3) The union of any family of prime $w$-ideals of $R$ is the union of a finite number of prime $w$-ideals of $R$. 
Proof. $(2) \Rightarrow(3)$ Assume that every GV-torsion-free $R$-module is a $w$-ZD $R$ module. Let $\left\{\mathfrak{p}_{\alpha}\right\}_{\alpha \in \Lambda}$ be any family of prime $w$-ideals of $R$. Let $M:=\bigoplus_{\alpha \in \Lambda} R / \mathfrak{p}_{\alpha}$. Then it is easy to see that $\mathscr{Z}(M)=\bigcup_{\alpha \in \Lambda} \mathfrak{p}_{\alpha}$. Note that $M$ is GV-torsion-free. By hypothesis, $M$ is a $w$-ZD $R$-module and so $\mathscr{Z}(M)=\bigcup_{i=1}^{t} \mathfrak{q}_{i}$ for some finite number of prime $w$-ideals $\mathfrak{q}_{1}, \ldots, \mathfrak{q}_{t}$ of $R$. Thus $\mathscr{Z}(M)=\bigcup_{\alpha \in \Lambda} \mathfrak{p}_{\alpha}=\bigcup_{i=1}^{t} \mathfrak{q}_{i}$.

$(3) \Rightarrow(2)$ Assume that the union of any family of prime $w$-ideals of $R$ is the union of a finite number of prime $w$-ideals of $R$. Let $M$ be any GV-torsionfree $R$-module. Let $N$ be a $w$-submodule of $M$ with $N \neq M$. Note that $R \backslash \mathscr{Z}(M / N)$ is a saturated multiplicatively closed subset of $R$. Hence by [12, Theorem 2], $\mathscr{Z}(M / N)$ is a union of prime $w$-ideals of $R$. By assumption it follows that $\mathscr{Z}(M / N)$ is the union of a finite number of prime $w$-ideals of $R$. Thus $M$ is a $w$-ZD $R$-module.

$(3) \Rightarrow(1)$ We first prove that if $R$ satisfies (3), then $R$ has only a finite number of maximal $w$-ideals. Let $\left\{\mathfrak{m}_{\alpha}\right\}_{\alpha \in \Lambda}$ be the family of all maximal $w$ ideals of $R$. By hypothesis, $\bigcup_{\alpha \in \Lambda} \mathfrak{m}_{\alpha}=\bigcup_{i=1}^{s} \mathfrak{q}_{i}$ for some finite number of prime $w$-ideals $\mathfrak{q}_{1}, \ldots, \mathfrak{q}_{s}$ of $R$. For each $i=1, \ldots, s$, let $\mathfrak{m}_{i}$ be a maximal $w$-ideal of $R$ such that $\mathfrak{q}_{i} \subseteq \mathfrak{m}_{i}$. Then it is clear that $\bigcup_{\alpha \in \Lambda} \mathfrak{m}_{\alpha}=\bigcup_{i=1}^{s} \mathfrak{q}_{i}=\bigcup_{i=1}^{s} \mathfrak{m}_{i}$. Now it is evident that distinct elements among $\mathfrak{m}_{1}, \ldots, \mathfrak{m}_{s}$ are all the maximal $w$-ideals of $R$.

Now if $\mathfrak{m}$ is a maximal ideal of $R$, then $\mathfrak{m} \subseteq \bigcup \mathfrak{m}_{i}$, where the union runs through all maximal $w$-ideals of $R$, since it follows from [22, Corollary 3.3 and Proposition 3.5] that $(r)_{w} \neq R$ for every nonunit $r \in R$. By the Prime Avoidance Theorem, we have that $\mathfrak{m} \subseteq \mathfrak{m}_{i}$ for some $i$, and so by the maximality of $\mathfrak{m}$, we have that $\mathfrak{m}=\mathfrak{m}_{i}$. Therefore every maximal ideal of $R$ is a $w$-ideal, and so $R$ is a DW ring, that is, every ideal of $R$ is a $w$-ideal.

$(1) \Rightarrow(3)$. This follows from $[17$, Proposition 1] and the fact that $\mathscr{Z}(M / N)$ is a union of prime $w$-ideals of $R$ for every proper $w$-submodule $N$ of a GVtorsion-free $R$-module $M$.

In [6, Theorem 1], it was shown that for a commutative ring $R$ with identity, the power series ring $R[[X]]$ in one variable over $R$ is Laskerian if and only if $R$ is Noetherian. In [16], Park showed that there exists an SM domain $R$ such that $R[[X]]$ is not an SM domain. Thus we have the following.

Question. For a commutative ring (or an integral domain) $R$, is it true that $R[[X]]$ is $w$-Laskerian if and only if $R$ is $w$-Noetherian?

Acknowledgements. The authors would like to thank the referee for a careful reading and many helpful suggestions which improve the paper. The first author was supported by the Basic Science Research Program through the 
National Research Foundation of Korea (NRF) funded by the Ministry of Education, Science and Technology (2010-0011996). The second author was financially supported by Changwon National University in 2013-2014.

\section{References}

[1] S. El Baghdadi, On a class of Prüfer v-multiplication domains, Comm. Algebra 30 (2002), no. 8, 3723-3742.

[2] S. El Baghdadi, H. Kim, and F. Wang, A note on generalized Krull domains, J. Algebra Appl. 13 (2014), no. 7, 1450029 (18 pp).

[3] E. G. Evans, Jr., Zero divisors in Noetherian-like rings, Trans. Amer. Math. Soc. 155 (1971), no. 2, 505-512.

[4] G. Fusacchia, Strong semistar Noetherian domains, Houston J. Math. 39 (2013), no. 1, $1-20$.

[5] R. Gilmer, Multiplicative Ideal Theory, Queen's Papers in Pure and Applied Mathematics, vol 90, Queen's University, Kingston, Ontario, 1992.

[6] R. W. Gilmer and W. Heinzer, The Laskerian property, power series rings and Noetherian spectra, Proc. Amer. Math. Soc. 79 (1980), no. 1, 13-16.

[7] J. R. Hedstrom and E. G. Houston, Some remarks on star operations, J. Pure Appl. Algebra 18 (1980), no. 1, 37-44.

[8] W. Heinzer and D. Lantz, The Laskerian property in commutative rings, J. Algebra 101 (1981), no. 1, 101-114.

[9] W. Heinzer and J. Ohm, Locally Noetherian commutative rings, Trans. Amer. Math. Soc. 158 (1971), no. 2, 273-284.

[10] - On the Noetherian-like rings of E. G. Evans, Proc. Amer. Math. Soc. 34 (1972), no. $1,73-74$.

[11] B. G. Kang, Prüfer v-multiplication domains and the ring $R[X]_{N_{v}}$, J. Algebra 123 (1989), no. 1, 151-170.

[12] I. Kaplansky, Commutative Rings, University of Chicago Press, Chicago 1974.

[13] H. Kim, Module-theoretic characterizations of t-linkative domains, Comm. Algebra 36 (2008), no. 5, 1649-1670.

[14] H. Kim and T. I. Kwon, Integral domains which are t-locally Noetherian, J. Chungcheong Math. Soc. 24 (2011), 843-848.

[15] H. Kim and F. Wang, On $\phi$-strong Mori rings, Houston J. Math. 38 (2012), no. 2, 359-371.

[16] M. H. Park, Power series rings over strong Mori domains. J. Algebra 270 (2003), no. $1,361-368$.

[17] S. Visweswaran, A note on universally zero-divisor rings. Bull. Austral. Math. Soc. 43 (1991), no. 2, 233-239.

[18] F. Wang, Finitely presented type modules and $w$-coherent rings, J. Sichuan Normal Univ. 33 (2010), 1-9.

[19] F. Wang and R. L. McCasland, On w-modules over strong Mori domains, Comm. Algebra 25 (1997), no. 4, 1285-1306.

[20] F. Wang and J. Zhang, Injective modules over $w$-Noetherian rings, Acta Math. Sinica (Chin. Ser.) $\mathbf{5 3}$ (2010), no. 6, 1119-1130.

[21] L. Xie, F. Wang, and Y. Tian, On w-linked overrings, J. Math. Res. Exposition 31 (2011), no. 2, 337-346.

[22] H. Yin, F. Wang, X. Zhu, and Y. Chen. w-Modules over commutative rings, J. Korean Math. Soc. 48 (2011), no. 1, 207-222. 
HWANKOO KIM

School of Computer and Information EngineERING

Hoseo University

ASAN 336-795, KOREA

E-mail address: hkkim@hoseo.edu

TAE IN KWON

Department of Mathematics

Changwon National University

Changwon 641-773, Korea

E-mail address: taekwon@changwon.ac.kr

Min Surp RheE

Department of Mathematics

DANKOOK UNIVERSITY

Cheonan 330-714, Korea

E-mail address: msrhee@dankook.ac.kr 